\title{
Integración, conciliación de políticas y diferencias de estructura económica
}

E.1 tema sobre el cual debo desarrollar mi ponencia parece y es de una extrema complejidad. Para comenzar, me gustaria delimitar el campo de esta ponencia indicando cómo veo yo el alcance del tema propuesto.

En primer lugar, desearía establecer la vinculación existente entre las dificultades estructurales $y$ coyunturales y las polfticas nacionales que deberán conciliarse con el esfuerzo de integración del Grupo Andino.

Las políticas nacionales pueden: a) emanar de un rango o elcmento de la estructura económica de un pais, ser una resultante de él o b) diseñarse deliberadamente para invalidar o atenuar o, alternativamente, reforzar sus consecuencias sobre la actividad económica. Un ejemplo de la primera circunstancia: el predominio de la producción minera y petrolera suele dar al Estado un mayor poder financiero $y$, por lo mismo, una mayor capacidad de iniciativa y acción directa de la que es usual cuando en la estructura económica predomina la agricultura. El uso de esa mayor capacidad de acción directa es así una resultante de un rasgo de estructura económica. Un ejemplo de la segunda circunstancia: en el caso de ese mismo país minero petrolero, la prioridad de una política de diversificación de la producción interna y de las exportaciones es un esfuerzo deliberado de atenuar el efecto del predominio de la mineria o el petróleo en la actividad económica.

Las características de estructura económica influyen pues doblemente sobre la política económica. La primera clase de influencia en una gran mayoría de los casos no es materia de opción. Su existencia está cleterminada por una característica o elemento de la estructura económica.

En el segundo caso esta deterninación no existe: la política se elige entre una serie de opciones posibles para reforzar o anular las consecuencias del rasgo de estructura. La misma integración económica es una de esas opciones en relación con algunos problemas de estructura 
del desarrollo económico, especialmente las deficiencias de la estructura industrial.

Con estos conceptos podemos formular varios juicios que nos sirven para delimitar el campo del estudio:

19) Cuando las políticas económicas resultan determinadas por una estructura económica nacional hay poco lugar a una conciliación de éstas con los instrumentos de la integración, a menos que esa estructura cambie. Mientras eso no suceda, en caso de conflicto con la integración, al menos en el corto y mediano plazo, ésta se hallará forzosamente limitada por tales políticas y las posibilidades de conciliación descansan más bien en una modificación de los instrumentos de la integración para adaptarse a ellos;

$\left.2^{9}\right)$ Cuando una política económica ha sido diseñada para reforzar o anular un. rasgo de estructura de una economía nacional y existe un conflicto con los instrumentos de la integración, usualmente habrá campo a la búsqueda de otras opciones no conflictivas de política $y^{\prime}$ hay, por lo mismo, lugar a una conciliación, en base sobre todo a una modificación de esa política, siempre que exista una motivación suficiente en el país cuya acción ha provocado el conflicto.

39) Cuando el conflicto con la integración es. el resultado de la elección de políticas alternativas a esa misma integración, como medios de anular o atenuar rasgos de la estructura económica de un país, como el libre cambio a la autarquía, existe una situación de incompatibilidad que no puede ser resuelta sino con el abandono de esa política conflictiva o con la renuncia a la integración.

De ese modo, creo que el tema de esta ponencia queda suficientemente acotado: si se trata de la primera clase de políticas nacionales, el esfuerzo del análisis debe centrarse en examinar los efectos que ellas pueden tener en la integración y cómo es posible adaptar los instrumentos de esa integración para conseguir los mejores resultados, al menos mientras los rasgos de estructura no cambien fundamentalmente. Es decir, se trata de una conciliación de la integración con respecto a esas politicas, lo que equivale a decir, respecto a los rasgos de estructura que las determinan. En el otro extremo, la tercera situación nos describe un caso en que no hay lugar a conciliación. La incompatibiliclad es por definición inconciliable, a menos que una de las partes ceda totalmente en su posición. La segunda situación, en cambio, caracteriza el campo en el cual es posible una conciliación mediante la elección de otras opciones de política, que sean teficaces para conseguir los fines propuestos y que no sean conflictivas con la integración. 
Tengo la impresión de que al establecer el tema de esta ponencia se pensaba sobre todo en esta última clase de políticas. Por ejemplo, si el instrumento elegido por un país para la promoción de exportaciones son las subvenciones o subsidios, que tarde o temprano entrarán en conflicto con la integración, los instrumentos de la integración, entre ellos el mismo arancel externo común, le darán la posibilidad de substituir ese sistema por otros que estimulen al productor sin crear condiciones inequitativas de competencia. En realiclad, toda la urdiembre "clásica" de los instrumentos de la integración constituye un marco que posibilita la búsqueda de opciones distintas a las políticas económicas nacionales $y$, en ese sentido, este tipo de conciliación es parte normal del proceso de integración.

Sin embargo, creo que no están entre estas conciliaciones los problemas realmente difíciles de la integración. Aunque cabe hacer una reserva: si las motivaciones a la integración están jerárquicamente subordinadas a las motivaciones sociales que llevan a las políticas conflictivas y las opciones que ésta ofrece o permite son, o se cree que son, menos eficaces para resolver el problema de estructura de que se trate, la conciliación puede volverse imposible y en ese sentido Ilegar a incompatibilidades asimilables a las señaladas en la segunda situación. Por ejemplo, una politica de fomento de la inversión extranjera adoptada por un país puede crear situaciones de conflicto con el esfuerzo de integración por las ventajas que ella puede dar al mismo sobre los otros miembros del Grupo para atraer inversión extranjera. Esa situación llevará a una fórmula de conciliación que puede ser un régimen común. Si el país valora más las ventajas que puede traerle una política de estímulo a la inversión extranjera que aquelias que puede derivar de la integración con el régimen común, existe una situación de incompatibilidad de hecho, ya no de esencia, como ia que antes comentamos, pero que tiene efectos similares en cuanto a la inviabilidad de toda conciliación. Es en definitiva un problema de jerarquía de motivaciones sociales. Otro ejemplo, creo que aclarará aún más estas situaciones: una integración que intente combinar economías capitalistas con economías socialistas llegará más o menos rápidamente a incompatibilidades de este último género. Toda integración supone necesariamente una apertura de mercado parcial o total y la existencia de un mecanismo que regule las decisiones de vender y comprar, de asignar recursos. Si ese mecanismo es el sistema no interferido de precios, surgirá el conflicto con una organización económica socialista; si por el contrario son decisiones de planificación, la contradicción surgirá para los países capitalistas. No habrá conciliación posible sin un cambio parcial o total de los sistemas que los acerque en el uso de estos instrumentos básicos. Si, 
como es probable, los Gobiernos valoran más sus sistemas políticos que las alternativas que les ofrece la integración, surgirá nuevamente una situación de incompatibilidad de hecho que, para distinguirla de la primeramente descrita, que denominamos esencial, la calificaremos como funcional.

Los dos ejemplos presentados parecerian constituir un mentís a la afirmación anterior que decía que no estaban entre estas conciliaciones, las de la segunda situación. los problemas más difíciles de la integración. Me atrevería sin embargo, a sostener que esa afirmación sigule siendo válida, porque si se observ'a con cuildado los dos problemas citados se llegará a la conclusión de que la razón de la imposibilidad de la conciliación no es la impotencia de la fórmula de integración para ofrecer opciones, sino la inferior jerarquía de las motivaciones a la integración frente a aquellas que han originado las politicas conflictivas.

Ya que se trata de valores o preferencias sociales, entramos de lieno en un problema de política $y$ es interesante observar que los dos casos de incompatibilidad señalados: la esencial citada al principio, y la funcional que se acaba de describir, tienen un marcado carácter ideológico. La tesis, tan cleseable, del pluralismo ideológico tiene a esa luz, una validez muy limitada en el caso de la integración ya que es funcional solamente dentrss de fronteras relativamente estrechas que enmarcan la posibilidad de una conciliación, que es, al final, la substancia del proceso político de toda integración.

Los razonamientos hechos nos permiten ya concluir esta sección que trataba de orientarse en el campo de estudio. Con la excepción de aquellos conflictos que están ideológicamente determinados, los problemas que se suscitan en la integración cuando existen opciones, muchas veces ofrecidas por la propia integración, a las políticas conflictivas destinadas a cambiar o reforzar rasgos de estructura de las economías que se integran, no suelen constituir los más difíciles obstáculos al proceso de integración. En realidad, son la materia normal del proceso de integración. Los problemas más serios aparecen cuando la integración debe conciliar políticas que están determinadas por rasgos de estructura que ella no puede modificar, o no puede modificar en plazo relevante. Ese creo que es el campo de real interés para el análisis, porque en el fondo nos lleva al tema más delicado de la integración: ¿cómo ésta puede conseguirse frente a la heterogeneidad de estructuras económicas de los países que participan en ella, ya que esas políticas, tal como se han definido, no son al final sino manifestaciones de las distintas estructuras económicas que se esfuerzan por integrarse? Se advertirá que no he tocado hasta ahora el problema de la coyuntura. Sin negar su importancia, 
crucial en los momentos difíciles, lo considero un tema de interés secundario frente a los tratados hasta ahora.

Dedicaré, por lo mismo, estas breves reflexiones a los problemas de conciliación derivados de las diferencias de estructura económica de los paises que constituyen una agrupación de integración. No menciono ya las diferencias de política, porque, como antes se ha discutido, las que más nos interesan entre estas politicas son las que existen como derivación, de una estructura económica que las determina. Es más lógico, por lo mismo, volver ese análisis hacia su origen: las propias estructuras. Hablo además de diferencias, por que estos conflictos surgen en la misma medida en que hay heterogeneidad de estructura entre los paises que constituyen la agrupación de integración.

La raiz de estos problemas difíciles de la integración lo veo asj en la heterogeneidad de estructuras económicas entre los paises que se integran. Esa no es una variable examinada explicitamente en las teorías "clásicas" de la integración, excepto cuando se analizan los efectos de creación o desviación de comercio en la integración de economías complementarias o competitivas. Sin embargo, para mí tiene una importancia clave, entre otras cosas porque detrás de esa variable yace ese elemento inmanente a toda relación social, especialmente si hablamos de relaciones internacionales, que es la relación de poder, poder económico y político.

Al poner el énfasis en la heterogeneidad, parto en realidad de una hipótesis que es necesario hacer explicita: mientras más homogéneos sean los paises en su estructura económica (y política, podría decirse), más viable y menos conflictivo será el esfuerzo de integración entre ellos, es decir más apto será el sistema para conciliar Ias aspiraciones o motivaciones nacionales con las del conjunto. Creo que es asi porque, en primer lugar, la relativa homogeneidad de estructuras se traduce en una homogencidad de motivaciones o preferencias zonales hacia la integración, y en segundo lugar porque la homogeneidad, al reflejarse en las políticas cconómicas y sociales con parecida jerarquización de objetivos e instrumentos, hace más coherente y menos conflictivo el proceso de integración.

La heterogeneidad de estructuras tendrá el efecto contrario y mientras mayor ella sea, más conflictivo será el proceso de integración, lo que equivale a decir, que más dificil será conciliar las preferencias y objetivos nacionales con lo de la zona integrada. La ponencia tendrá pues como tema central el análisis de la heterogeneidad $y^{\prime}$ sus efectos sobre la integración. Esta heterogeneidad 
se referirá a las estructuras económicas, sin que ello niegue que igual trascendencia pueden terier heterogeneidades de otro orden especialmente los de carácter político. El análisis se iniciará con conceptos de carácter general, y sólo más tarde, cuando el marco conceptual haya sido planteado, nos referiremos concretamente al caso del Grupo Andino.

Como supuesto previo, aclararé que para quien esto escribe la motivación social $y$, en esa medida, el objetivo fundamental de la integración entre países en desarrollo es el cambio de la estructura industrial. La integración puede tener y tiene otras motivaciones y objetivos de carácter económico o político, pero para los paíscs subdesarrollados - por lo mismo con superficiales y deficientes estructuras industriales - la motivación básica es pasar, gracias a la ampliación del espacio económico, a un nivel distinto de desarrollo industrial con una estructura más integrada, más capaz de transmitir su dinamismo a las otras actividades económicas. Es a la luz de esta motivación y objetivo que trataré de estudiar los problemas de conciliación que emergen de la heterogeneidad.

Para simplificar el análisis, creo que es conveniente dividir los elementos de estructura que pueden ser origen de diferencias relevantes al problema de integración en dos categorías. La primera categoría se refiere directamente a las diferencias del grado de desarrollo industrial alcanzado. La segunda categoría abarca a los otros elementos de la estructura económica que pueden originar dificultades en la armonización de políticas necesarias para crear el espacio ampliado sobre el cual debe asentarse el cambio esperado de la estructura industrial.

Dadas las complejidades de medir el grado de desarrollo industrial, de encontrar un patrón común de medida, he optado por una variable que creo representativa de las diferencias en el desarrollo industrial; ésta es la diferencia en. la dimensión económica entre los países, entendida como la capacidad de los países como productores y como mercado, capacidad que se refieja, con todas las limitaciones conocidas, en el ingreso o producto nacional, el PIB total, más bien que el PIB o el ingreso por habitante. Diríamos así que a mayor dimensión económica de un país corresponde un mayor grado de desarrollo industrial, o si se quiere una estructura industrial más moderna e independiente. Este aserto parece corresponder bastante con la realidad, especialmente en el caso de la América Latina, cuyos países han seguido desde hace varias décadas políticas más o menos sistemáticas de sustición de importaciones, que se han fundado en un aprovechamiento pertinaz de las oportunidades de actividad industrial que les proporcionaba su mercado nacional. 
Puede haber desviaciones dadas por una sobreutilización o una subutilización de ese mercado nacional, inducidas, especialmente, por el grado de protección ${ }^{3}$, pero esas desviaciones tenderán a corregirse con el tiempo. En la perspectiva histórica, podría clecirse que es estrecha la correlación existente entre la dimensión económica de un país así entendida, y su grado de desarrollo industrial. Un ejemplo de sobreindustrialización, o si se quiere, sobreutilización del mercado, estaría dado por Chile, al menos el Chile anterior a 1979. Un ejemplo posible de subutilización, es posible el de Venezuela. Lo que importa es que, en los dos casos, habría o hay una conciencia real de su dimensión económica, del potencial de su mercado y es esa percepción la que se ha reflejado en los objetivos que esos dos paises han buscado en la integración y sus actitudes frente a la necesidad de conciliación de política. ${ }^{2}$.

Podríamos decir así que en esta primera categoría de diferencias de estructura, la diferencia relevante es la dimensión económica entre los países que se integran. Sin dar los detalles de un análisis de esta variable que cs muy engorroso nos limitaremos a señalar que teóricamente las condiciones más propicias a la integración existen cuando:

a) Menor dimensión. económica tienen los países que se integran.

b) Mayor es la dimensión económica de la zona de integración.

c) Más homogéneos son los países que se integran en cuanto a su dimensión económica.

Hay otros elementos que intervienen en esta conclusión y es menester aclararlos. Ella supone, creo que razonablemente, que mientras más pequeño es un país en cuanto a dimensión económica, mús motivado se halla a la integración como un medio de vencer sus limitaciones internas a la industrialización. Esa motivación social le lleva a aceptar, más fácilmente que otros mayores, las limitaciones a su actuar autónomo que puede significar la conciliación de politicas. Del mismo modo, mientras mayor sea la zona de integración, más precisamente, mientras más se acerque éste al rango de dimensión que, dado el estado de la técnica, se considere requerido para el desarrollo industrial moderno, más lugar tienen a manifestarse las เI! :"

1Lo que implica que existc un "óptimo" en la protección dentro de una tcoŕa dinámica de desarrollo. El concepto es tan complejo que no cabría intentar su formulación en esta ponencia.

EEvidentemente antes del cambio de posición de Chile al elegir una opción distinta a la integración como ámbito para el desarrollo de las actividades. 
motivaciones a la integración de los países que se integran. En lo que se refiere a la tercera condición, la de la homogeneidad, basta decir que ella tencierá a hacer más coherente en intensidad y jerarquización las motivaciones de los países que se integran. Es más fácil que haya, en ese caso, consenso sobre los objetivos e instrumentos de la integración y es mayor el ámbito posible de conciliación de politicas. Si estas tres condiciones se dan, es más viable la integración económica, y ella puede ser a la vez profunda y amplia.

Las condiciones más propicias no son, sin embargo, un patrón útil para confrontarlo con la realidad. Una de esas condiciones, la homogeneidad, aun entendida laxamente, es lo opuesto a la norma de la naturaleza y la sociedad que es la heterogeneidad. Como método, es pues más fructífero analizar las desviaciones del caso ideal.

Sobre esas bases, las condiciones menos propicias a la integración se darán cuando:

a) Mayor dimensión económica tengan los países que se integran.

b) Menor es la dimensión económica de la zona de integración.

c) Más heterogéneos son los países que se integran en cuanto a dimensión económica.

Después de las explicaciones anteriores es poco lo que se puede añadir sobre las condiciones a) y b); en cambio conviene ahondar un poco en los efectos de la heterogeneidad de dimensiones. La diferencia de dimensión económica, como representativa de grados distintos de desarrollo inclustrial, tiene un doble efecto: en primer lugar, un efecto absoluto: una diferencia de motivaciones, en su intensidad y jerarquización, entre los países que se integran; lo podríamos calificar como una "incoherencia" de motivaciones. Un país de gran dimensión económica estará menos motivado por la integración de un país pequeño y será, en consecuencia, menos propenso a ceder posiciones en una conciliación de políticas que un país menor. Por lo mismo, serán distintos tanto los objetivos que los dos buscan en. la integración como los instrumentos que cada uno considera de prioridad. Pero ese no es el único efecto. El segundo, que podríamos llamarlo "efecto relativo" de la diferencia de dimensión económica, resultará de la posición en que se, encuentre cada país en cuanto a dimensión económica frente a los otros miembros de la zona de integración. Importa en este caso las modificaciones que sufren sus motivaciones a la integración en razón de las diferencias que existen con los otros miembros de la zona de integración. Ellas pueden ser debido a dos hechos: a) Una diferencia real en cuanto al a provechamiento 
de las oportunidades de industrialización: un país de mayor dimensión económica normalmente habrá aprovechado en su política de importaciones muchas de las oportunidades industriales que el pais pequeño busca en la integración, como una etapa lógica de su evolución industrial; y b) una diferencia virtual o potencial: por la misma razón anterior, el país de mayor dimensión económica estará en mejores condiciones, tendrá una mayor capacidad, para aprovechar las oportunidades que la integración le ofrezca. Hay una superioridad o inferioridad reales, una relación de poder económico, y lo que es políticamente más relevante, "sentimientos" colectivos de superioridad o inferioridad que son los que más afectan las motivaciones a la integración.

La incoherencia de motivaciones inherente a las diferencias de dimensión se verá así reforzada por estas reacciones emanadas de la posición relativa. En el caso extremo, de grandes diferencias de dimensión económica, y de poder, la integración se vuelve imposible. Es la razón por la cual no creo ni económica ni políticamente viabie para los países subdesarrollados la tesis del libre cambio, que es una integración en el mercado mundial donde reina la más extrema heterogeneidad. Es la razón por la cual en el caso del CAME, con la Unión Soviética como miembro, a pesar de todas las afinidades políticas, ha sido imposible llegar a una integración profunda y amplia. Sin llegar a los extremos, creo que es razonable admitir que una integración viable requerirá que las diferencias de dimensión económicas entre los países que se integran no sean tan grandes que cierren real o "psicológicamente" las posibilidades de cambio industrial de cuales. quiera de los países que sean menores que otros. Aun asi, como se verá, existen problemas, pero eso parece ser una primera condición de viabilidad.

Conviene ahora examinar más sistemáticamente las situaciones intermedias entre los dos extremos dados por las condiciones más y menos propicias. Para abreviar podría comenzarse eliminando de una en una las circunstancias propicias y examinando su efecto sobre la integración. Con las varias combinaciones posibles podrían así caracterizarse distintas situaciones, algunas de las cuales no permitirian ninguna forma de integración, excepto una cooperación bilateral muy limitada, mientras que otras podrian dar lugar a modelos típicos de integración que variarían en cuanto a objetivos e instrumentos, es decir, en su profundidad y amplitud. Para el caso del Grupo Andino interesa examinar únicamente una de esas situaciones que considero la relevante a sus circunstancias reales.

Cabría decir que el Grupo Andino no está muy Iejos de las dos primeras condiciones propicias: en primer Iugar, todos los países que 
lo constituyen, a pesar de sus diferencias de dimensión económica, están intensamente motivados por la posibilidad del cambio industrial que les ofrece el mercado ampliado, que es el resultado que importa en 'esa primera variable; existirán' grados en esa intensidad, pero en mi opinión son realmente matices de una preferencia social que les es común. En ese sentido no pueden ser calificados como países grandes, como, sin duda, será el caso de Brasil. En segundo lugar, 1a. integración es de una dimensión suficiente para permitir, dentro de límites de ineficiencia razonables, ese cambio de la estructura industrial que creo es el objetivo esencial que todos buscan en la integración.

En cambio, dadas las posiciones relativas, puede afirmarse que el Acuerdo de Cartagena está constituido por un grupo heterogéneo de países en cuanto a su dimensión económica. Creo que esa heterogeneidad no llega a los extremos antes mencionados que harían inviable toda integración; pero es, de todos modos, una circunstancia adversa que haría muy dificil la integración o conduciría a una integración superficial y laxa si el Grupo no hubiera encontrado un mecanismo de compensación de esa heterogeneidad que, generalizando, es el único modo de proponerse una integración profunda $y$ amplia cuando prima esa heterogeneidad, aun cuando las otras dos condiciones estén tan cerca del óptimo como pueda concebirse.

En el Grupo Andino, evidentemente, no puede afirmarse que ninguna de esas condiciones sean "óptimas" y algunos problemas de conciliación pueden derivarse de incoherencia de motivaciones que se traduzca en posiciones relativamente distintas sobre los objetivos e instrumentos de la integración. Ejemplos tenemos de esas dificultades. Sin embargo, como dije, no creo que esté alli el problema mayor de esta primera categoría de conciliación de las diferencias de estructura. Aparte de diferencias ideológicas insalvables que eventualmente pueden presentarse, el problema mayor del Grupo Andino es la heterogeneidad de dimensión económica, lo que equivale a decir, de desarrollo industrial y, en otra esfera, de poder económico. Ese problema, por un rasgo de intuición política de quienes lo idearon, que yo me atrevería a llamar "genial", cuenta con un mecanismo de compensación que al menos en principio, parece adecuado: la programación industrial.

Estoy consciente que el análisis no ha añadido nada nuevo a la teoria informal que el Grupo Andino ha, sido decantado en el curso de su construcción. Personalmente, el discumir de estas ideas me reafirma, primero, en la importancia vital de que ese mecanismo de compensación de la heterogeneidad del Grupo funcione y sea un rasgo permanente del proceso de integración. Su existencia hace 
posible la primera y más difícil conciliación de las diferencias de estructura, las que resultan del distinto grado de desarrollo industrial, conciliación que ninguna otra fórmula de integración había podido conseguir hasta ahora, con las consecuencias lógicas sobre la profundidad y amplitud de la integración o sobre su propia existencia. Si nos empeñamos realmente en que el mecanismo de compensación exista y funcione, estoy convencido, en segundo Iugar, que el Grupo es una fórmula de integración intrinsecamente viable. Los otros problemas de conciliación pueden ser bastante difíciles, como luego se verá brevemente, pero este primero es el más delicado y complejo. Pareceria que vamos a salvarlo felizmente.

Una vez examinada esta primera categoría de diferencias de estructura, aquellas que se resumen en la dimensión económica, nos resta por revisar el efecto de las heterogeneidades que se originan en otros rasgos de esas estructuras económicas. Por limitaciones de tiempo no será posible ni tampoco oportuno analizar toda la gama de diferencias de estructura que son teóricamente significativas para la integración. Hay forzosamente que limitarse a unas cuantas que se consideren más relevantes a las circunstancias del Grupo Andino. In ellas ni siquicra cabe profundizar hasta el punto de anticipar todos los problemas posibles y dar las fórmulas de solución. Ello sería no sólo pretencioso, sino desconsiderado para este estudio. Me limitaré a señalar los problemas más claros de conciliación y, en algunos casos, sugerir líneas posibles de acción para enfrentarlos.

Para esta exposición seguiré el mismo marco de análisis desarrollado en relación con las diferencias de dimensión económica. En el modelo de integración que tan simplificadamente hemos trazado, la heterogencidad que surge de las diferencias de estructura que vamos a mencionar tiene efectos similares a los ya señalados en el caso de la dimensión. Aun cuando las otras dos condiciones sean. propicias, es decir, tamaño de los paises y de la zona de integración, si existe una heterogeneidad en determinados rasgos de esa estructura la integración tendrá que adaptarse a ella, modificando sus objetivos de profundidad y amplitud, a menos que se encuentren mecanismos de compensación que permitan anular o atenuar el efecto de esas diferencias. También, como en el caso anterior, existirán extremos de heterogeneidad que no pueden ser contrarrestados por ningún mecanismo y que señalan las fronteras de una integración viable, al menos de una integración formal que se proponga objetivos ambiciosos.

Mencionaremos esos elementos de estructura según la secuencia en 
que es posible que los probleams consiguientes se manifiesten en el proceso de integración.

\section{ESTRUCTURA DEL COMERGIO EXTERIOR}

Acostumbrados a la gravedad endémica de los problemas de balanza de pagos de los países subdesarrollados, se puede pasar por alto que pueden existir entre ellos diferencias muy marcadas en relación con Ia estructura del comercio exterior; creo que el Grupo Andino es un buen ejemplo de las mismas.

Para empezar, no para todos los paises subdesarrollados tiene la misma probabilidad el riesgo del llamado estrangulamiento externo. Creo en el fondo que todo país en desarrollo, si no mejora significativamente su cstructura productiva, caerá tarde o temprano en un estrangulamiento de balanza de pagos ya sea por el déficit comercial o por el déficit de la balanza de capitales. Pero ese "tarde o temprano" hace toda la diferencia. Para unos países el riesgo es siempre inminente. Para otros es una sombra en el futuro que a menudo se ignora en la política de corto y mediano plazo. Un buen ejemplo actual son los grandes países petroleros.

Además, la estructura del comercio exterior, puede estar más o menos marcada por el signo de la inestabilidad y esa puede ser una de las razones, no la única, por las cuales está más presente en las perspectivas el riesgo del estrangulamiento externo. Ello dependerá de la naturaleza de los principales productos de exportación y del grado de control que los paises exportadores ejerzan sobre el mercado. Casos hay -nuevamente el petróleo- en que existe efectivamenite un cierto control del exportador primario sobre el precio.

Cuando el signo ha sido la inestabilidad, la diversificación de las exportaciones -en ese caso, un camino sin alternativas- puede realmente forzar al país a buscar deliberadamente políticas menos protectoras, que discriminen menos contra las exportaciones, sobre todo si tienen ya peso en la composición de éstas los productos industriales. No se trata de las tesis extremas a las que nos acostumbraron las últimas vicisitudes del Grupo Andino, sino de posiciones realistas que emanan de la lógica de una evolución gradual de Ias estructuras económicas.

Finalmente, para no alargar esta lista de variables que pueden dar lugar a heterogeneidades en cuanto a la estructura del comercio exterior, podría añadirse el de la mayor o menor dependencia del 
abastecimiento nacional en la importación de alimentos y materias primas o productos intermedios; determinante del empleo, los últimos, y muy ligada a las bases de toda política económica y social, la primera.

Aunque se puede esperar que buena parte de esos rasgos cambien con la propia integración, que en realidad, está hecha para contribuir a remediarlos, no se puede ignorar que la integración toma tiempo, actúa lentamente, y por lo pronto esas heterogeneidades de estructura están presentes, surten efectos importantes y será grave error no tomarlas en cuenta como hechos persistentes en las políticas de integración. Por sus efectos, unos países buscarán un mayor grado de apertura externa que otros. Algunos necesitarán más y estarán más acostumbrados que otros, a usar con flexibilidad instrumentos como el tipo de cambio o los subsidios. Por tradición, además, el empleo de esos instrumentos tendrá mayor aceptación política por parte de la comunidad. Otros países, más expuestos a la inestabilidad, insistirán en contar como reserva con ciertos instrumentos de rápida aplicación y de efecto cierto para alterar el curso de las importaciones, como las restricciones cuantitativas.

Las dificultades de conciliar todas esas derivaciones de estructuras distintas del comercio exterior y la balanza de pagos tenderán a concentrarse básicamente en la negociación del arancel externo común, la armonización de los instrumentos para-arancelarios y las políticas cambiarias y, más limitadamente, en el manejo de las cláusulas de salvaguardia. Teóricamente se pueden proponer instrumentos que reemplacen con ventaja a todo ese confuso arsenal de que los paises se valen para sobrerivir: su racionalidad económica puede ser evidente pero si por razones de estructura, ellos afectan de distinto modo a los paises o no responden a lo que parece ser políticamente viable dadas las situaciones reales y las expectativas, en resumen, si ignoran que hay una diferencia de estructuras que tardará bastante tiempo en modificarse, lo más probable es que ni siquiera sean considerados. La conciliación será todavía más complicada si varía significativamente entre los paises Ia importancia del comercio intrarregional frente al comercio total.

No quiero alargarme respecto a las vías de solución porque, entre otras cosas, no tengo la pretensión de conocerlas. Sólo quisiera insistir en que es probable que, al menos temporalmente, la conciliación pueda hacerse si en lugar de buscar las formas perfectas se trata de ir en etapas en la armonización de los instrumentos. El Arancel Externo Común no será perfecto si se admite la existencia de instrumentos para arancelarios temporalmente no armonizados, pero será un instrumento aplicable. Es posible además que se dilate en el 
mercado y los precios ha sido recogida como un instrumento básico. Se la interfiere con decisiones deliberadas de localización, mediante la programación industrial; pero aun en esa circunstancia, el mercado sigue siendo el mecanismo esencial para determinar precios $\mathrm{y}$ montos en las transacciones. En esas condiciones, la heterogeneidad mencionada es, o puede llegar a ser, un problema serio.

Una parte del problema, la menor, se resolveria con la programación que reserva campos de acción a todos los países, campos que no pueden ser invadidos por un Estado vecino más acucioso. La otra, la fundamental, aquella que se relaciona con el comportamiento del Estado ante el mercado, tendria arreglo si se pudiera comprometerlo a responder a los estímulos del mercado en la misma forma en que lo hace un sujeto de economía privada. Veo difícil alcanzar una solución de esa clase que sea funcional. Si esto no es posible o no lo fuera totalmente, la alternativa o complemento sería un estatuto especial que establezca genéricamente las obligaciones del Estado dentro del proceso de integración, obligaciones que en casos extremos sólo podrían ser efectivas a través de un proceso de programación y negociación desarrolladas, v. g. para establecer cantidades de compra de determinados bienes, precios de transacciones, etc. El tema tiene gran importancia y en verdad no ha sido hasta ahora resuelto satisfactoriamente en procesos entre países en desarrollo. Lo ha sido en el CAME, pero en ese caso no se trata de un proceso de integración que pueda calificarse como profundo y amplio, ni es una integración que se realiza entre economías mixtas en desarrollo.

No creo que en las circunstancias actuales del Grupo Andino las diferencias sean tales que requieran soluciones heroicas. Pueden crecer en el curso del tiempo a medida que se avance en el desarrollo de la industria de integración que, por su misma complejidad, originará todo un abanico de diferencias en la participación estatal. Creo, por lo mismo, que es hora de preocuparse del tema e ir gradualmente diseñando los rasgos del mecanismo apropiado de compensación. Es tarea delicada que exigirá imaginación y un sentido práctico. Es bueno saber que ninguna de las dos cualidades le ha faltado nunca al Grupo Andino.

\section{LA XNFLAGIÓN}

He m'encionado antes que, inevitablemente tendrá que llegarse a una armonización de las politicas cambiarias si se quiere dar profundidad y amplitud a la integración. Lo he afirmado pensando, sobre todo, 
que en sus efectos inmediatos sobre el comercio, el tipo de cambio es uno de los pocos mecanismos efectivos de compensación de las diferencias en el grado de inflación de los países miembros de un esquema de integración.

No quisiera entrar a discutir aquí el origen y la naturaleza de nuestras inflaciones. Creo sí que, cuando son endémicas, ellas marcan de tal modo la estructura de la actividad económica que, sus consecuencias pueden bien considerarse como un rasgo de estructura relevante a la integración, en el sentido en que antes hemos usado ese concepto. La inflación es pues el origen de heterogeneidades persistentes que pueden poner límites a los alcances de los procesos de integración, si de algún modo no se hallan mecanismos de compensación a sus efectos inmediatos sobre el comercio y, a más largo plazo, si no se elimina gradualmente el grado de heterogeneidad dado por la coexistencia de distintas tasas de inflación. Es uno de los casos en que el remedio final no puede venir sino de una modificación de esos rasgos heterogéneos de estructura, a la cual sin duda puede contribuir la propia integración.

Los efectos más sensibles de la inflación sobre el comercio, más precisamente, sobre la relación de los precios internos con el exterior, pueden ser compensados, con todas las limitaciones conocidas, mediante devaluaciones frecuentes. Ese ha sido el método habitualmente seguido por varios países del Grupo Andino. Por hallarse el Grupo en su etapa formativa, ese sistema ha cumplido su función y no se han advertido efectos graves sobre las corrientes de comercio. Por supuesto, los países que han preferido mantener fijo el tipo de cambio en este período de inflación generalizada han sufrido con esas políticas y es posibie que la situación ya se refleje de modo significativo en las cifras de exportación. El instrumento para compensar esa situación está por supuesto disponible: la devaluación. Circunstancias estructurales, como las mencionadas al referirse al comercio exterior, vinculadas a una tradición política difícil de modificar en corto o mediano plazo, puede dificultar el uso de este instrumento. Si el problema adquiere magnitud, y esi probable que asi sea en el curso de los próximos años, se tendrá que dar la alternativa de algún instrumento substitutivo. Nada más que como un ejemplo mencionaré uno vinculado a una armonización de las subvenciones a la exportación clentro de la subregión que es, sin duda, una tarea urgente. Dentro de esa armonización podría autorizarse a los países que, por las circunstancias indicadas, no están en condiciones de modificar el tipo de campio para neutralizar el efecto de la inflación interna, a establecer subvenciones adicionales al nivel armonizado en Ia cuantía necesaria para conseguir ese efecto. Los cos- 
tos de esa medida para el país que la use lo inducirán a emplear con el tiempo el instrumento más eficaz: el tipo de cambio; pero al menos en el corto plazo no tendrá que enfrentar un deterioro creciente en su comercio dentro del Grupo, sin duda un elemento crítico en esta etapa de construcción. Una medida de esa clase es, sin duda, discutible desde muchos puntos de vista. Podría producir distorsiones serias si se prolonga. La he mencionado sólo como un ejemplo del esfuerzo que hay que hacer para encontrar alternativas cuando el óptimo no es alcanzable.

No obstante su importancia, este temprano efecto sobre el comercio es el problema menor derivado de las diferencias de inflación entre los países miembros de un proceso de integración. Existen otras derivaciones que son mucho más dificiles de contrarrestar $y$, aun cuando más tardías en manifestarse, pueden acentuar todavía más agudamente la heterogeneidad entre los países. Son las distorsiones que la inflación produce en los precios relativos $y$, en general, en el sistema de asignación de recursos. A medida que la integración aranza y se profundiza, esta consecuencia más general de la inflación se hará sentir cada vez más pesadamente si hay diferencias grandes entre los países en ese sentido. No existe, por supuesto, otro medio para resolver el problema que abordar gradualmente la armonización de las políticas cambiarias y monetarias cuando el objetivo de la integración es ambicioso. Si esa armonización es imposible por razones de otro orden, la realidad impondrá pronto límites al proceso de integración. Ello no significa que cualquier integración se torna infactible; pero sí que habría que reducir el alcance de sus objetivos cuando éstos implicaban un proceso profundo y amplio. Un ejemplo de la persistencia de este obstáculo, es lo que sucede ahora en la Comunidad Económica Europea cuando ha tenido que enfrentar tendencias diferentes de inflación y problemas de balanza de pagos como consecuencia de una coyuntura internacional que ha afectado en grados distintos a sus países miembros.

Finalmente quisiera mencionar brevemente otras causas de heterogeneidad a las que no se suele dar la debida consideración, porque no se espera que sus manifestaciones aparezcan pronto en el horizonte de la integración o porque se intuye que su tratamiento será por fuerza extremadamente conflictivo si se aborda prematuramente. No obstante, creo que es mejor tener clara la realidad a la que nos enfrentaremos si la integración vence estas etapas iniciales de construcción. 
ESTRUCTURA Y POLÍTICAS SOGIALES, ESPECIALMENTE EN RELACIÓN CON LA PROPIEDAD DE LOS FACTORES DE LA PRODUCCIÓN, LA GESTIÓN DE LA EMPRESA. Y LA DISTRIBUCIÓN DEL INGRESO

La coexistencia en una fórmula de integración de países con diferencias notorias en su estructura social y en sus políticas sociales puede traducirse, con el paso del tiempo, en conflictos serios dentro del proceso de integración. Se trata de una heterogeneidad delicada que puede desencadenar reacciones negativas de sectores que, de otro modo, habrian sido favorables o al menos indiferentes a la integración. Las reacciones pueden surgir de las dos partes. Los países con una estructura social más evolucionada y, por ejemplo, salarios más altos, tendrán recelo de una apertura de mercados en la cual llevarian una desventaja al menos en ciertas actividades.

Las condiciones de propiedad y gestión, si ellas han sido objeto de reformas para modificar su estructura, es posible que sean una causa de incertidumbre que se refleje en dificultades para aprovechar las oportunidades de la integración. Por su parte los paises menos adeIantados socialmente, o al menos los grupos que se benefician con el status quo, resistirán armonizaciones de políticas que puedan afectar sus intereses y traer cambios de importancia en la situación social. En cambio, los países más avanzados tratarán de acelerar esas armonizaciones de politica para al menos atenuar las diferencias que les parecen menos favorables, desde el punto de vista de los costos y los incentivos de la inversión.

En conjunto, el efecto es tornar más conflictivo el uso de muchos de Ios instrumentos de la integración, entre ellos la propia apertura de mercados y una serie de armonizaciones de políticas en el terreno económico y social. Las diferencias tienen prolongaciones más complejas en el terreno de los incentivos de la inversión. Según las circunstancias, uno u otro país se podrá sentir en cierta inferioridad de condiciones de competencia para inducir inversiones en relación con los otros. En ese caso, tendría razones para desconfiar de la asignación a través del mecanismo de mercado e insistir en la inclusión de mecanismos de programación formal, tan detallados como sea posible.

Como se ve, aun excluyendo los extremos ideológicos, un pluralismo político de amplio espectro puede constituir una limitación importante para la factibilidad de los esfuerzos de integración. Si las diferencias son muy acentuadas, los mecanismos para corregir 
la heterogeneidad son mús complejos y muy probablemente haya que restringir el alcance $y$ la profundidad de la integración, especialmente en cuanto a las amonizaciones más generales de políticas, lo que no deja de repercutir en todo el conjunto del proceso de integración.

Si se quiere dar a la integración toda la profundidad de que es capaz, la solución es, por supuesto, eliminar las diferencias de estructura y de políticas sociales mẹdiante una armonización gradual, que es realmente una aproximación de modelos políticos. Tratar de hacerlo fróntalmente $y$ antes de que la integración haya realmente echado raices sería ir al fracaso. En tales condiciones no es nada probable que la motivación a la integración sea suficiente para conseguir resultados en esferas que tocan dinectamente los intereses de los grupos detentadores del poder, ya que se supone que el sentido de esa armonización no puede ser regresivo con respecto a la situación del más evolucionado. Pero es, asimismo, evidente que más pronto o más tarcle estaremos ante la disyuntiva: o detener la 'integración' mucho antes de alcanzar las metas económicas elevadas que ésta se había propuesto o abordar, con prudencia pero con resolución, esta armonización a la postre clave de estructuras y sistemas politicos.

Creo que he agotado la paciencia de los queridos lectores amigos con esta exposición árida đe los problemas de la integración. Un tema se me ha quedado en el tintero: el de la conciliación de políticas frente a la coyuntura económica. Sé que es muy importante. pero he preferido por ahora concentrarme en los aspectos que a mí me parecen los limitantes más serios de la integración. Pido disculpas por esa omisión.

Dije antes que esta ponencia habia sido escrita con aridez, una aridez que ustedes han soportado con bondad. Quizás lo hice así porque mientras más hondo cala un tema en la emoción, más duro debe ser uno consigo mismo para mirarlo con el despego que vexige la objetividad. Véase esa aridez mía como un síntoma de cuán cerca estä este tema de mis vivencias más entrañables. 